\title{
Demonstration of lesions of cerebral toxoplasmosis by computerized tomography
}

\author{
Geoffrey P. SUMmerfield \\ M.A., B.M., B.Ch., M.R.C.P. \\ Department of Haematology, University of Liverpool, Royal Liverpool Hospital
}

\begin{abstract}
Summary
A patient who was treated for 11 years with cytotoxic drugs for Hodgkin's disease developed cerebral toxoplasmosis. Discrete lesions including an occipital abscess were visualized in the brain by computerized tomography (CT). This permitted a brain biopsy to be taken but the appearances were non-specific. At post-mortem pseudocysts of Toxoplasma gondii were found in several sites within the CNS. The recognition of these CT appearances as being due to toxoplasmosis should in future prompt serological investigation and urgent treatment of this potentially curable condition, despite negative biopsy material.
\end{abstract}

\section{Introduction}

Cerebral toxoplasmosis is being increasingly recognized as a significant cause of morbidity and mortality in immunosuppressed patients, especially in those with Hodgkin's disease (Carey et al., 1973; Ruskin and Remington, 1976). Such a patient is now described in whom cerebral lesions, proved at post-mortem to be due to toxoplasmosis, had been demonstrated in vivo by computerized tomography (CT).

\section{Case report}

A 48-year-old woman was first seen in the Royal Liverpool Hospital for review in October 1971. In 1967, she had developed generalized lymphadenopathy and hepatosplenomegaly with weight loss, night sweats and malaise. A lymph node biopsy showed Hodgkin's disease and she was considered to have Stage IVB disease on clinical grounds. She was treated with injections of vinblastine alone, in doses of 1-2 mg fortnightly.

In October 1974, the patient was transferred to the care of the haematology department. She continued with her injections of vinblastine. In August 1975, she developed subcutaneous nodules on her arms and left thigh, and her voice became hoarse. A skin biopsy was taken which suggested infiltration by Hodgkin's disease. A deposit was seen on the larynx. The chemotherapy was changed to bleomycin, cyclophosphamide, vinblastine and prednisone in fortnightly courses, which produced resolution of the skin lesions and the hoarseness after 3 courses. Over the next 2 years, repeated courses of combined chemotherapy, consisting of a variety of drugs, were required in order to contain the disease.

In November 1977 she became deaf in both ears, worse on the left side, and developed headaches. These improved but in December she became deaf again, with sore throat, fever and white sputum. There was a purulent discharge from both ears. She was treated with antibiotics and the discharge cleared but she remained tired. In mid-Decembes she was admitted with a history of confusion an⿺廴⿻肀二 restlessness for 2 days, with vague frontal an occipital headaches. There was flattening of affect with disorientation in time and space, amnesia, disinhibition and mental confusion. There was a coarse nystagmus, secondary to the right and primary to the left; the left pupil was larger than the right, there was a mild left facial weakness and bilateral hearing loss, worse on the right side. She veered to the left when walking. There was no neck stiffness. Lumbar puncture showed WBC $16 / \mathrm{mm}^{3}$ (all lymphocytes), no $\mathrm{RBC}$, protein $>100 \mathrm{mg} \%$, 흥 globulin slightly increased, CSF glucose 2.73 . $\mathrm{mmol} / \mathrm{l}$. Skull X-ray was normal. Isotope brain $\dot{\sigma}$ scan was normal, but CT revealed multiple small $\stackrel{3}{-}$. bilateral lesions in the basal ganglia and brain stem, of and a large right-sided occipital lesion with the appearance of an abscess (Figs 1 and 2). Biopsy 욱 of this lesion produced gelatinous material but no $>$ pus. There was non-specific glial proliferation on histology.

The CT appearances were not interpreted at that $\sigma$ time as being due to cerebral toxoplasmosis, $\mathcal{O}$ especially as the biopsy was negative. The patient $\underset{\omega}{N}$ deteriorated with increasing confusion, rambling $\underset{<}{\sigma}$ speech and a tendency to wander around the ward. In mid-January 1978 , she became rapidly worse and died. At post-mortem, examination of the brain $\stackrel{\oplus}{?}$ showed multiple foci of gliosis throughout the $T$ cerebral cortex and thalamus. An area of gliosis 


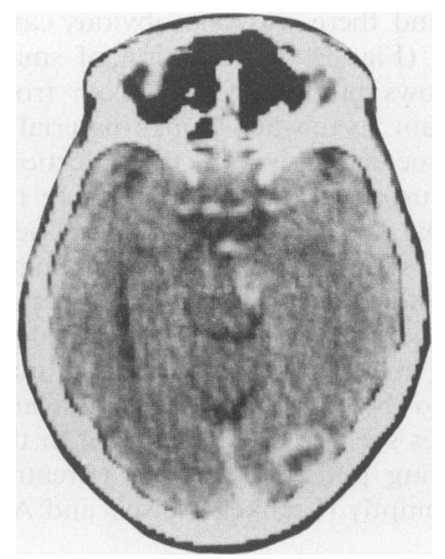

FIG. 1. CT scan showing Toxoplasma lesions in brain stem and ring lesion (representing an abscess) in right occipital lobe.

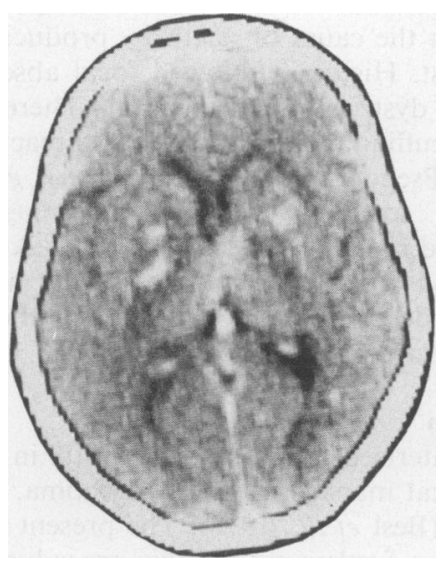

FIG. 2. CT scan showing Toxoplasma lesions in basal ganglia.

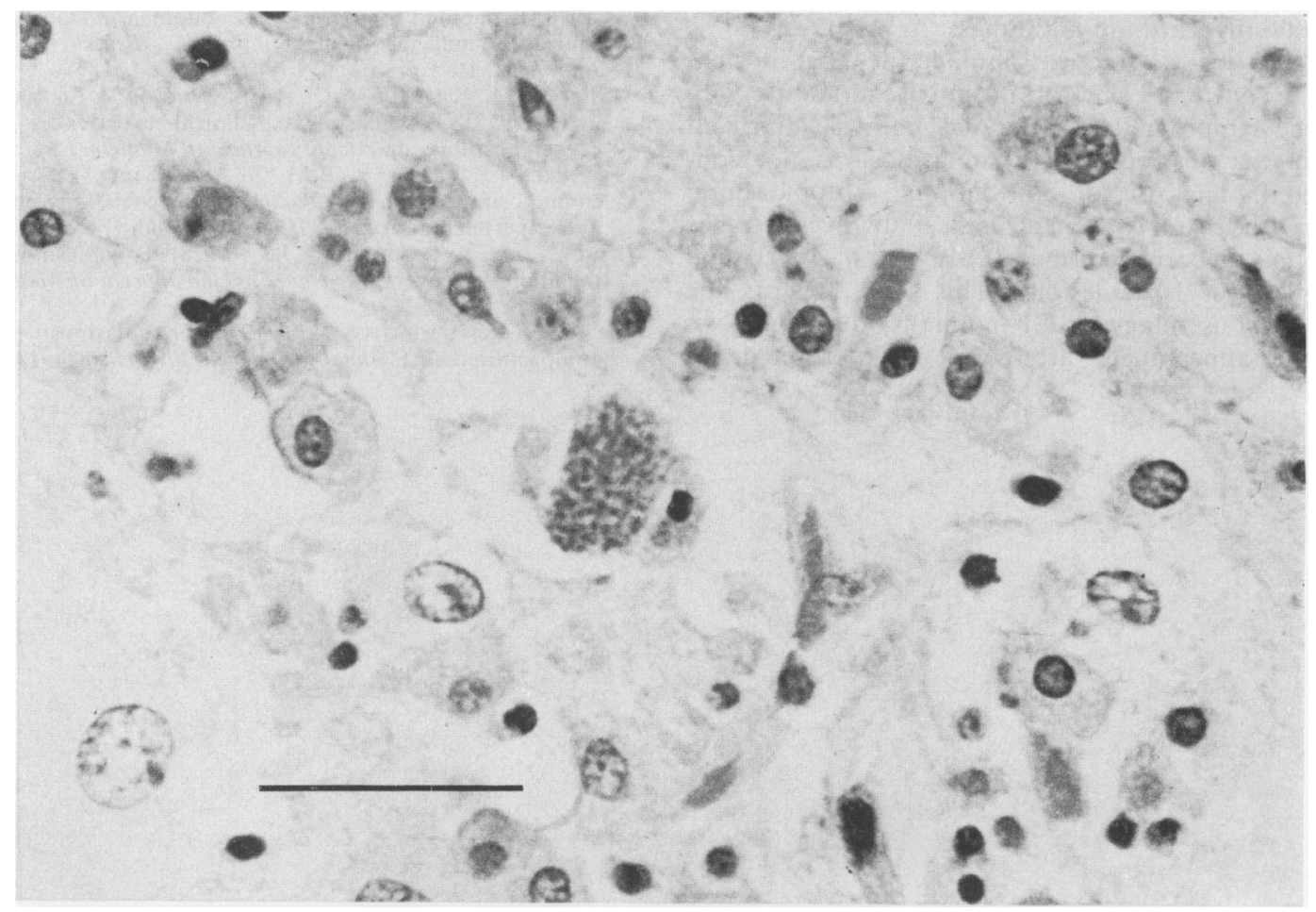

Fig. 3. Photomicrograph showing Toxoplasma cyst in brain substance (scale marker equals $50 \mu \mathrm{m}$ ). 
was also found in the medulla oblongata and may have been the cause of death by producing respiratory arrest. Histology showed focal abscess formation and dystrophic calcification. There was perivascular cuffing by lymphocytes and reactive macrophages. Pseudocysts of Toxoplasma gondii were found in sections from the thalamus, occipital cortex and medulla (Fig. 3). The Hodgkin's disease was found to be in remission, with no enlarged lymph nodes and no involvement of the spleen or bone marrow.

\section{Discussion}

Computerized tomography is useful in the staging and clinical management of lymphoma, as recently reported (Best et al., 1978). The present case report illustrates a further use for this procedure in Hodgkin's disease.

Symptomatic, often fatal, toxoplasmosis occurs more commonly in Hodgkin's disease than in any other malignant condition (Carey et al., 1973; Ruskin and Remington, 1976). This is due to chronically impaired cell-mediated immunity resulting both from the disease itself and from the drugs used in its treatment. In these circumstances, $T$. gondii most often affects the brain, diffusely or locally, myocardium and lungs.

Diagnosis is frequently made at post-mortem (Carey et al., 1973; Ruskin and Remington, 1976) as the symptoms and signs may be misleading and often the possibility of toxoplasmosis is not considered. The use of conventional isotopic brain scans in cases with neurological involvement has previously been described (Carey et al., 1973) and may detect large lesions. This case suggests the potential usefulness of CT in ante-mortem diagnosis if the appearances are correctly interpreted. In this patient the lesions were focal, discrete and $\frac{2}{\Phi}$ scattered and there was one obvious cavity due to $\stackrel{\unrhd}{\varrho}$ an abscess (Fig. 1). Localization of small cerebral $C$. lesions allows biopsies to be taken from affected $\underset{\vec{s}}{\vec{\rho}}$ areas of brain. Even when biopsy material is obtained by these means, however, the infection must be clinically suspected in order to ensure that appro- $\frac{O}{\omega}$ priate preparations (with Wright and Giemsa rather $\frac{\sigma}{\sigma}$ than HE stain) (Krick and Remington, 1978) are made and a careful search for organisms carried $\infty$ out. Even then, histology may not provide the correct $\overrightarrow{0}$ diagnosis. In these circumstances, it may be very valuable to be aware of the significance of CT $\vec{\omega}$ appearances such as those described in this patient, $\frac{\text { o }}{8}$ thus enabling potentially curative treatment to be started promptly (Frenkel, Nelson and Arias-Stella, 1975).

\section{Acknowledgments}

The author would like to thank Professor A. J. Bellingham $\vec{N}$ for permission to report this case and Dr L. Brock for his 0 generous assistance in producing the illustrations.

\section{References}

Best, J.J.K., Blackledge, G., Forbes, W.StC., Todd, I.D.H., Eddleston, B., Crowther, D. \& Isherwood, I. (1978) Computed tomography of abdomen in staging and clinical management of lymphoma. British Medie्ئl Journal, 2, 1675.

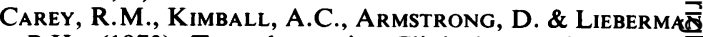
P.H. (1973) Toxoplasmosis. Clinical experiences in cancer hospital. American Journal of Medicine, 54, 30.

Frenkel, J.K., Nelson, B.M. \& ARIAs-Stella, J. (1975) Immunosuppression and Toxoplasma encephalitis: clinical and experimental aspects. Human Pathology, 6, 97.

KRICK, J.A. \& Remington, J.S. (1978) Toxoplasmosis in the adult - an overview. New England Journal of Medicine, 298, 550.

Ruskin, J. \& Remington, J.S. (1976) Toxoplasmosis in the compromised host. Annals of Internal Medicine, 84, 193. 